\title{
GLETSCHERSEEN
}

\section{GEDANKEN ZUR KLASSIFIKATION MIT BESONDERER BERÜCKSICHTIGUNG VON BEISPIELEN AUS DEN SCHWEIZERALPEN}

\section{Wolfgang Schweizer}

Mit 5 Figuren

Am 29. Juli 1956 war das Gadmental Schauplatz eines Naturereignisses, das leicht hätte zu größern Schäden führen können. Der Gletschersee beim Hotel Stein entleerte sich innert weniger Stunden und ließ das Gadmerwasser hoch ansteigen. Glücklicherweise waren keinerlei Folgen zu verzeichnen, weshalb denn auch der Vorgang in der Öffentlichkeit beinahe unvermerkt blieb. Für den Hydrographen und Geographen hingegen bedeutete er den Hinweis auf eine Naturerscheinung, die vermehrte Aufmerksamkeit verdient. Es handelt sich um die Entleerung eines Gletscherrandsees, welche zusammen mit verwandten Erscheinungen deshalb im folgenden dargestellt werden soll.

Die Seen als solche sind in der Erdgeschichte im ganzen recht veränderliche, kurzfristige Gebilde. Dies gilt erst recht für diejenigen unter ihnen, die ihr Dasein dem momentanen Stand und der Beschaffenheit eines Gletschers verdanken. Nach Rавот (Lit. 9) sind Gletscher-Randseen von den Wasseransammlungen auf dem Eis zu unterscheiden. Die nachfolgenden Ausführungen betreffen vor allem die Randseen.

Sie sind in drei Hinsichten von Interesse:

a) Geomorphologisch als Resultat der abtragenden Kräfte einerseits, der Stauwirkung des Eises oder der jüngsten Moränen andererseits.

b) Hydrologisch stellen sie das Ergebnis komplexer Wechselwirkungen von festem und flüssigem Niederschlag, Temperatur und Strahlung, Gletscherbewegung und Topographie dar. Ihr Wasserhaushalt wird weitgehend von der Schnee- und Gletscherschmelze beherrscht.

c) Anthropogeographisch bedeuten sie für die Talschaften flußabwärts oft latente Risiken, haben sich doch einmalige oder wiederholte Entleerungen solcher Seen durch Breschen im Eis oder in Moränen verschiedenenorts von alten Zeiten bis zur Gegenwart immer wieder ereignet.

Bild 1 zeigt den Gletschersee im Zungenbecken des stark zurückgeschmolzenen Steingletschers am Sustenpaß nach einer Aufnahme vom 28. Juli 1956. Der See nimmt darin den Platz ein, der noch vor zwanzig Jahren vom Eis der Gletscherzunge ausgefüllt war. Die Endmoräne bildet in mehr oder weniger deutlichem, hufeisenförmigem Wall den Abschluß nach der Talseite.

Die Darstellung der meteorologischen Verhältnisse der letzten Julitage 1956 gibt die Erklärung, wie es zum Ausbruch, d. h. zur raschen Entleerung dieses Sees kommen konnte. Am Abend des 27. Juli entlud sich ein Unwetter über dem Gadmental, wobei in Gadmen etwa eine Stunde lang grober Hagelschlag registriert wurde. In der Höhe der umliegenden Berge blieben Hagelkörner und Neuschnee bis zum folgenden Tag liegen. Die Wärme des klaren Sommertages ließ am 28. von allen Hängen Schmelzwasser herunterrinnen, und als ein neuer Gewitterregen niederging, stieg der Gletschersee am 29. so stark an, daß die Moräne an der Abflußstelle angegriffen wurde. Dies verstärkte den Abfluß; der See begann, sich zu entleeren, und die Moräne als lockere Aufschüttung vermochte der Strömung nicht zu wiederstehen. Das Gadmerwasser stieg während einiger Stunden zu bedrohlicher Höhe an und verstärkte von Innertkirchen an auch die Aare, doch wurden keine Schäden gemeldet.

Ein ähnlicher Fall ereignete sich während des Hochwassers Ende August 1954 im Oberengadin. Schauplatz des Seeausbruchs war das hintere Rosegtal, wo sich früher die Zungen des Tschierva- und des Roseggletschers vereinigt hatten. Der Rückzug brachte es mit sich, da $\beta$ heute zwei getrennte Gletscherenden vorliegen, wobei ein kleiner See eine Vertiefung vor dem Roseggletscher ausfüllte, teils durch Endmoränen, 


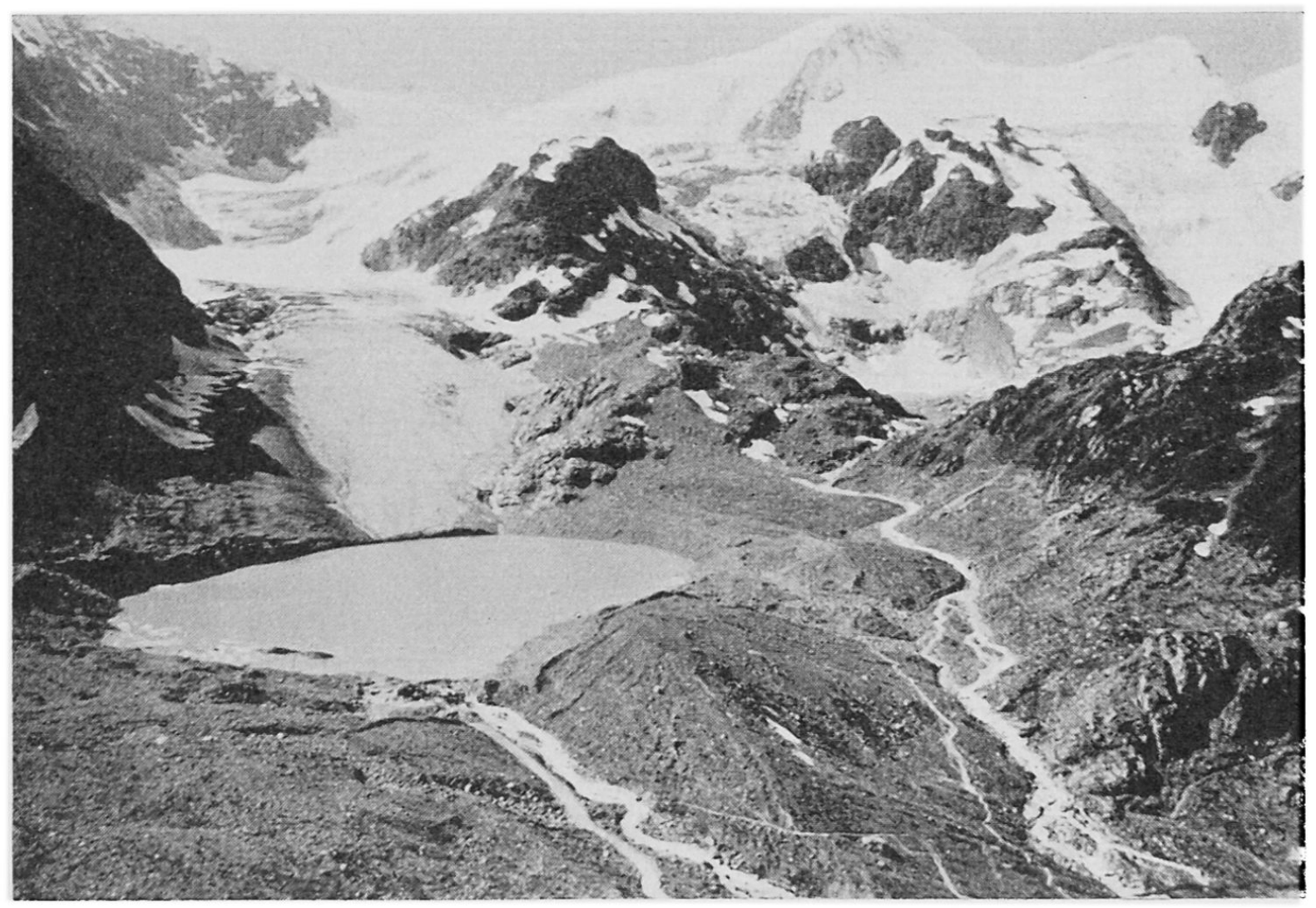

Abb. 1 Schmelzwassersee am Steingletscher, aufgenommen am 28. Juli 1956 kurz vor dem Ausbruch Photo IV. Schweizer

teils durch eine etwas höhere Mittelmoräne des früheren Zustandes abgedämmt. Der Überlauf des Sees stieg am 21./22. August 1954 infolge wolkenbruchartiger Niederschläge derart an, daß sich der Ablauf quer durch die Moräne stark vertiefen konnte. Durch diese Bresche, die stellenweise wie ein Cañon aussieht, gelangte in kurzer Zeit eine zusätzliche Wassermenge von etwa hunderttausend Kubikmetern mit viel Geschiebe talabwärts in den Inn und trug in hohem Maße zu den Schäden an Brücken, zu Dammbrüchen und Überflutungen bei (vgl. Lit. 10 und Abb. 2).

Aus den vergletscherten Anden von Peru werden verschiedene Seen beschrieben (Lit. 4 und 6), die sich zwischen einer mächtigen Endmoräne und der kleiner gewordenen Gletscherzunge gebildet haben. Einige davon sind von HeIm mit instruktiven Luftaufnahmen belegt. Das Beispiel der Laguna Cohup erscheint in Bezug auf Gestalt, Hydrologie und Wirkung ${ }^{1}$ auf die Umgebung derart typisch, daß man füglich von einem Gletschersee «Typ Cohup» sprechen kann. Seine Merkmale sind: Ansammlung von Schmelzwasser im Zungenbecken eines schwindenden Gletschers; einmaliger Durchbruch des Abflusses an einer Stelle der Endmoräne, verbunden mit katastrophaler Flut talauswärts. Ursache des Ausbruchs ist meist ein ungewöhnlicher Andrang von Schmelz- und Regenwasser; in andern Fällen kann er von einem großen Eissturz in den See ausgelöst werden. Zurück bleibt das mehr oder weniger leere Zungenbecken mit der zerteilten Moräne (Bild 3).

Wenn dieser Fall unter hunderten von im Rückzug begriffenen Gletschern nur in begrenzter Zahl vorkommt, so hängt dies damit zusammen, daß die Endmoräne nicht so oft genügend groß und zusammenhängend für die Bildung eines geschlossenen Beckens ist.

1 Die Laguna Cohup verursachte am 13. Dezember 1941 die katastrophale Flut, der ein Drittel der Stadt Huaras mit sechstausend Menschen zum Opfer fiel. 
Abb. 2 Durchbruch des Rosegbaches durch die Moräne nach der Entleerung des Gletschersees vom 22.8.1954. Aus TöNDURY G. A. "Ursachen und Bekämpfungsmöglichkeiten der zunehmenden Hochwassergefahr im Engadin *. Wasser- und Energiewirtschaft Nr. 12, 1954.

Photo O. Bisaz, Samedan

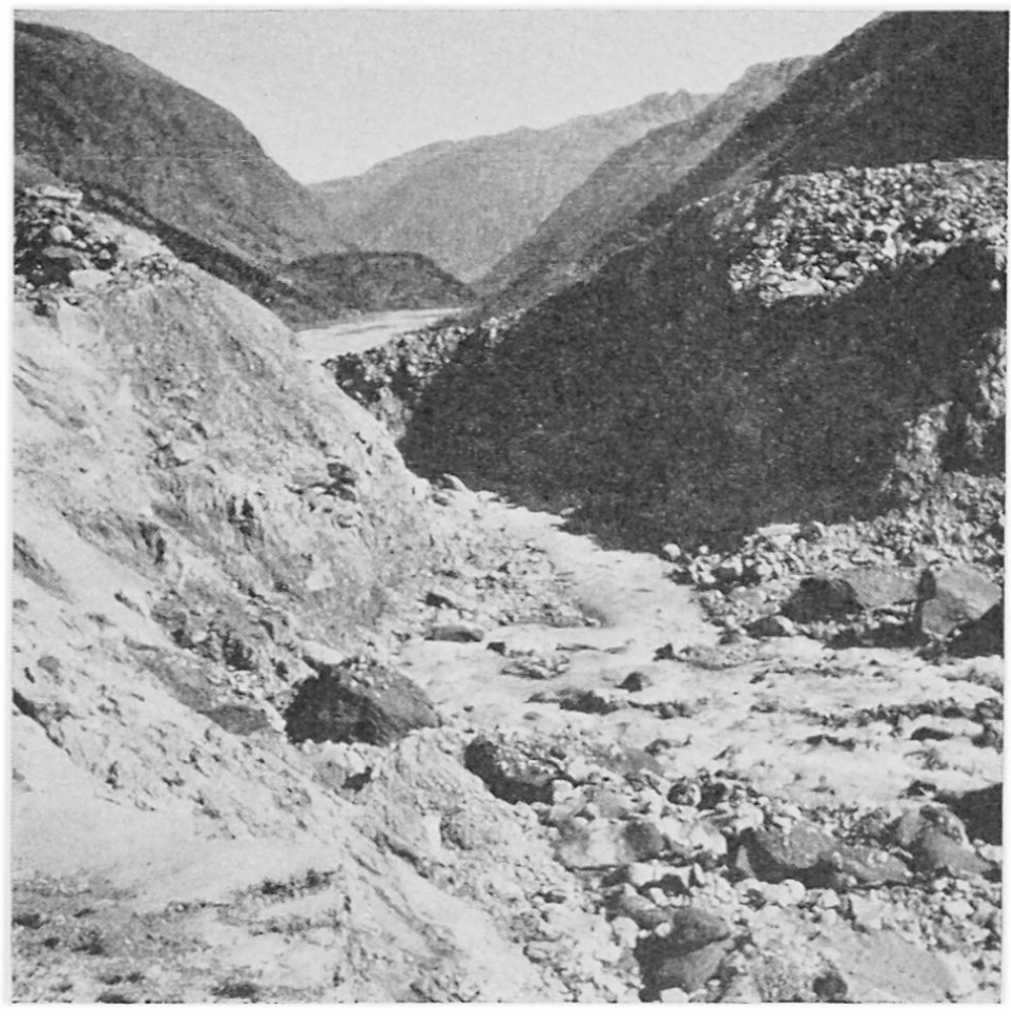

Ein Gletschersee anderer Art war der vom Allalingletscher gestaute Mattmarksee im hinteren Saastal. Bild 4 zeigt, daß heute anstelle des früheren Sees eine von verästelten Wasserläufen durchzogene Anschwemmungsebene vorliegt. Die Höhenlage dieser Ebene wird von der rechten Seitenmoräne ${ }^{2}$ des Allalingletschers bestimmt. Der Gletscher selbst reichte bis etwa 1925 als Eisbarriere quer ins Haupttal; seither erfolgte der starke Rückzug bis zur gegenwärtigen Lage des Eisrandes $300 \mathrm{~m}$ über dem Tal. Es ist offensichtlich, daß ein eigentlicher Gletscher-Stausee nur in Zeiten großer Ausdehnung des Eises vorhanden sein konnte. In Übereinstimmung mit den Nachrichten über die Maximalstände der Alpengletscher in historischer Zeit wird der Mattmarksee in Urkunden aus dem 15.-19. Jahrhundert häufig als Ursache furchtbarer Wasserschäden im Wallis genannt.

Aus der reich dokumentierten Geschichte des Mattmarktsees (Lit. 8) sei erwähnt, daß im Laufe von vierhundert Jahren etwa dreißig Ausbrüche stattfanden, die größten am 4. August 1633, 17. September 1772 und am 27. August 1834. Meist waren vorher bei warmem Sommerwetter gewaltige Gewitterregen niedergegangen und hatten den See zum Steigen gebracht. Floß dann die Vispa brausend durch die Spalten der stauenden Gletscherzunge, so erweiterte die Strömung den Durchfluß und konnte damit den Ausbruch des Sees einleiten. Dann wurden Eisschollen, Felsblöcke, weiter unten auch Bäume, Brücken und Gebäude auf die wilde Talfahrt mitgerissen. Gegen 20 Mill. $\mathrm{m}^{3}$ Wasser konnten so in Saas, Visp und im Rhonetal extreme Hochwasser verursachen. Nach den Katastrophen schloß sich jeweils die Lücke im Eis infolge der Bewegungen des Gletschers.

Die heute sehr abgelegene Gegend von Mattmark wurde von den Wallisern in früheren Jahrhunderten viel begangen, weil der Saumweg über den Moropa $\beta$ nach Italien dem See entlang führte. So konnten Säumer, Kaufleute und Söldner, die von Italien her kamen, im Saastal Auskunft über den Stand des Sees geben. Die Saaser bemühten sich nicht nur, die im Mittel-

2 Rezente Moräne, erst nach dem Rückzug des diluvialen Talgletschers entstanden. Der Allalingletscher muß in der Eiszeit seine reiche Fracht an Findlingen und Moränenschutt auf den Talgletscher geschoben haben. So sind unzählige Stücke von Saussurit-Gabbro als Leitgestein des Rhonegletschers ins früher vergletscherte Gebiet mitgeführt worden. 


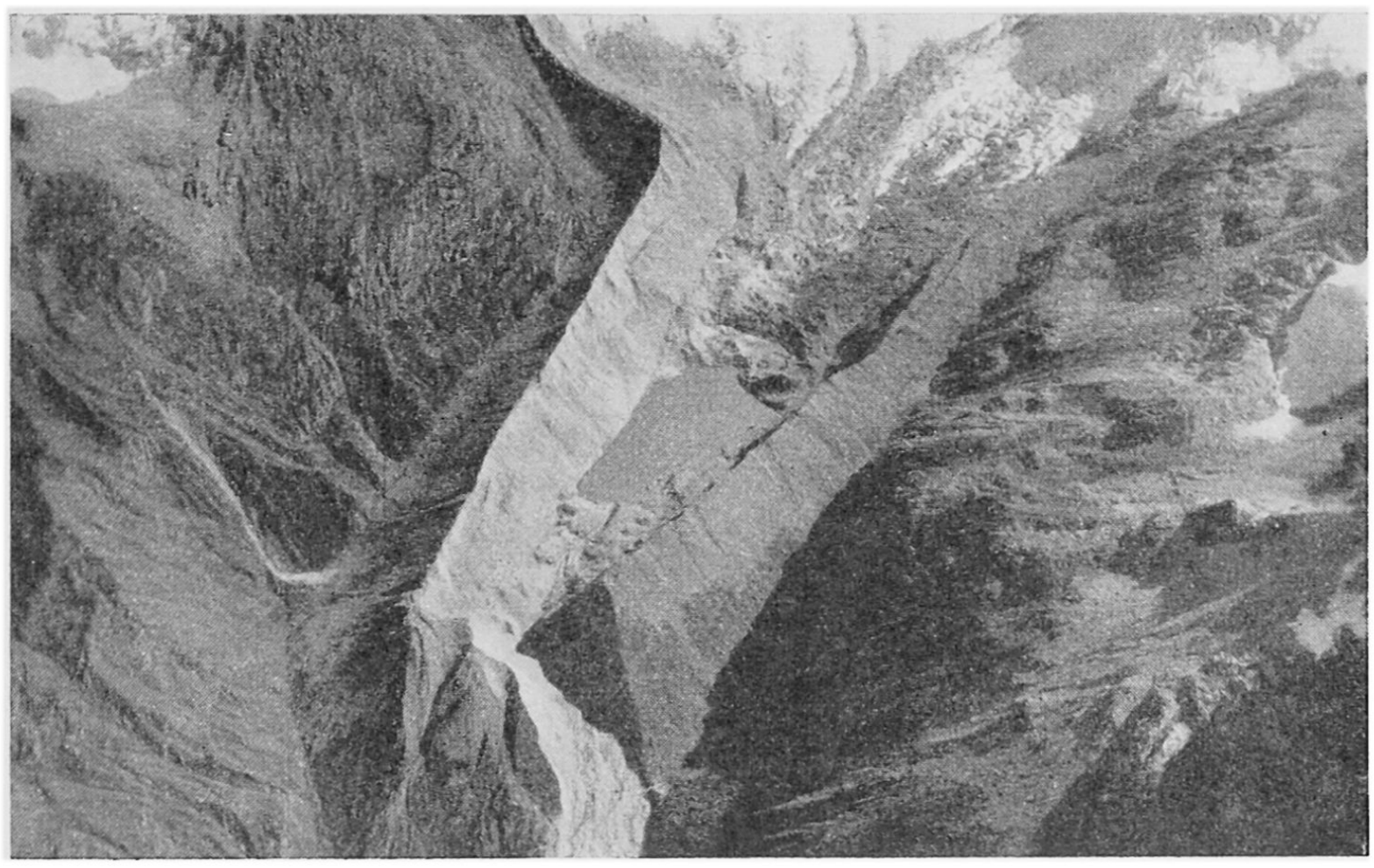

Abb. 3 Laguna Cohup in den Anden von Peru. Die Bresche in der Moı̈̈ne stammt vom Ausbruch am 13. Dezember 1941

Photo Arnold Heim 8.9.1947 Mit freundlicher Erlaubnis des Autors dem Buche allunderland Peru entnommen

alter gut gangbaren Saumwege trotz des Vorrückens der Gletscher instand zu halten; sie versuchten auch, die Gefahr des Gletschersees zu bannen. Bittschriften an die Geistlichkeit, ein $2 \mathrm{~m}$ tiefer, künstlicher Seeablauf und später ein künstlicher Stollen quer durchs Eis sollten Abhilfe bringen. Der Erfolg ließ jedoch zu wünschen übrig. Erst 1927 erfolgte die Ableitung der Vispa aus der Ebene von Mattmark durch einen $500 \mathrm{~m}$ langen Stollen im Fels des rechten Talhangs. Da der Gletscher als gefährlichstes Hindernis inzwischen jedoch verschwunden war, konnte der Stollen seinen eigentlichen $Z$ weck, die Umgehung der Gletscherzunge, bisher nie erfüllen. Er wird auch kaum mehr dazu kommen, da neuerdings die Erstellung eines künstlichen Mattmarksees mit einem großen Erddamm vorgesehen ist.

Es überrascht, da $\beta$ die breit angelegte Publikation über den Mattmarksee (Lit. 8) den Lac Combal im Quellgebiet der Dora Riparia nur mit einer halben Zeile erwähnt. Diese großartige Parallele zum Mattmarksee befindet sich unmittelbar südlich des Mont Blanc. Der See wird von der Moräne und der Gletscherzunge des Glacier de Miage italien gestaut. Die Notiz aus der zeitgenössischen Chronik von G. BÉrody, $\mathrm{da} ß \mathrm{am}$ 15. Juni 1620 im Aostatal eine Überschwemmung der Flüsse eingetreten sei, wobei die Wiesen und Weinberge großteils von Schutt bedeckt wurden, könnte sehr wohl auf einen Ausbruch des Lac Combal schließen lassen. Nach Lit. 1 ist ein großer Ausbruch des Lac Combal 1646 erfolgt.

Neben diesen zwei gleichartigen Gletscherseen in den Westalpen ist aus den Anden von Peru ein Fall von erstaunlicher Ähnlichkeit zu nennen: die Laguna Parrón (Lit. 4). Ich möchte für diese von Seitengletschern und ihren Moränen gestauten Seen im Haupttal die Bezeichnung «Typ Mattmark» vorschlagen. Sie zeichnen sich durch zviederholte Wasserausbrüche aus und werden bei Gletschervorstößen besonders gefährlich.

Nahe verwandt mit dieser Art sind die Fälle, da das Eis einer seitlichen Gletscherzunge im Haupttal Stauung bewirkt, ohne daß wesentliches Moränenmaterial beteiligt ist. Das eindrücklichste Beispiel dafür bot der Giétrozgletscher im Val de Bagnes. An der rechten Talflanke, hoch über dem steilen Hang ob Mauvoisin gelegen, bildete dieser Gletscher durch Abbrüche von Eis eine regenerierte Gletscherzunge 
im engen Tal unten. Kleinere Eismassen dieser Art konnte der Fluß, die Drance, schmelzen und wegführen. Im Frühjahr 1818 waren die Eisabbrüche jedoch derart voluminös, daß der enge Taleinschnitt auf eine Länge von einem Kilometer über hundert Meter hoch mit Eis erfüllt war. Mit dem Einsetzen der Schneeschmelze entstand ein Stausee (an der Stelle des heutigen Stausees von Mauvoisin), der am 16. Mai schon über $2 \mathrm{~km}$, am 13. Juni $3,6 \mathrm{~km}$ Länge besaß. Man war sich der großen Gefahr bewußt und hatte unter Leitung des Kantonsingenieurs Venetz einen Stollen durch das Eis getrieben, sodaß der See langsam auszulaufen begann. Seine Länge war so vom 13. bis zum 16., Juni auf $3 \mathrm{~km}$ zurückgegangen, als am Nachmittag jenes Tages die Eisbarriere brach und der Seeinhalt sich als furchtbare Flut ins Dranceund Rhonetal ergoß ${ }^{3}$. Für die Katastrophe, die etwa fünfzig Menschenleben forderte und fünfhundert Gebäude zerstörte, war jedenfalls die Schmelz- und Erosionswirkung des durch den Eisdamm strömenden Wassers ausschlaggebend. Gleiche Ereignisse hatten das Val de Bagnes schon 1595 und 1640 heimgesucht; sie wiederholten sich auch nach 1818, doch gelang es später, das stauende Eis durch zugeleitetes Quellwasser beizeiten $\mathrm{zu}$ schmelzen.

In den Ostalpen hat der Vernagtferner in gleicher Art zeitweise einen See im Haupttal der Rofner Ache gestaut. Dieser Rofensee ist in der Zeit von 1600 bis zur Mitte des 19. Jahrhunderts achtmal ausgebrochen, wobei bis zu 8 Mill. $\mathrm{m}^{3}$ Wasser innert einer Stunde wegflossen. (Die von einigen Autoren genannte Zahl von 60 Mill. $\mathrm{m}^{3}$ ist nicht zutreffend.) Für diese Stauwirkungen des Eises, die keine Auf-

3 Der $55 \mathrm{~m}$ tiefe See soll eine Wassermenge von 20 Mill. $\mathrm{m}^{3}$ innert eine halben Stunde ins Tal entleert haben.

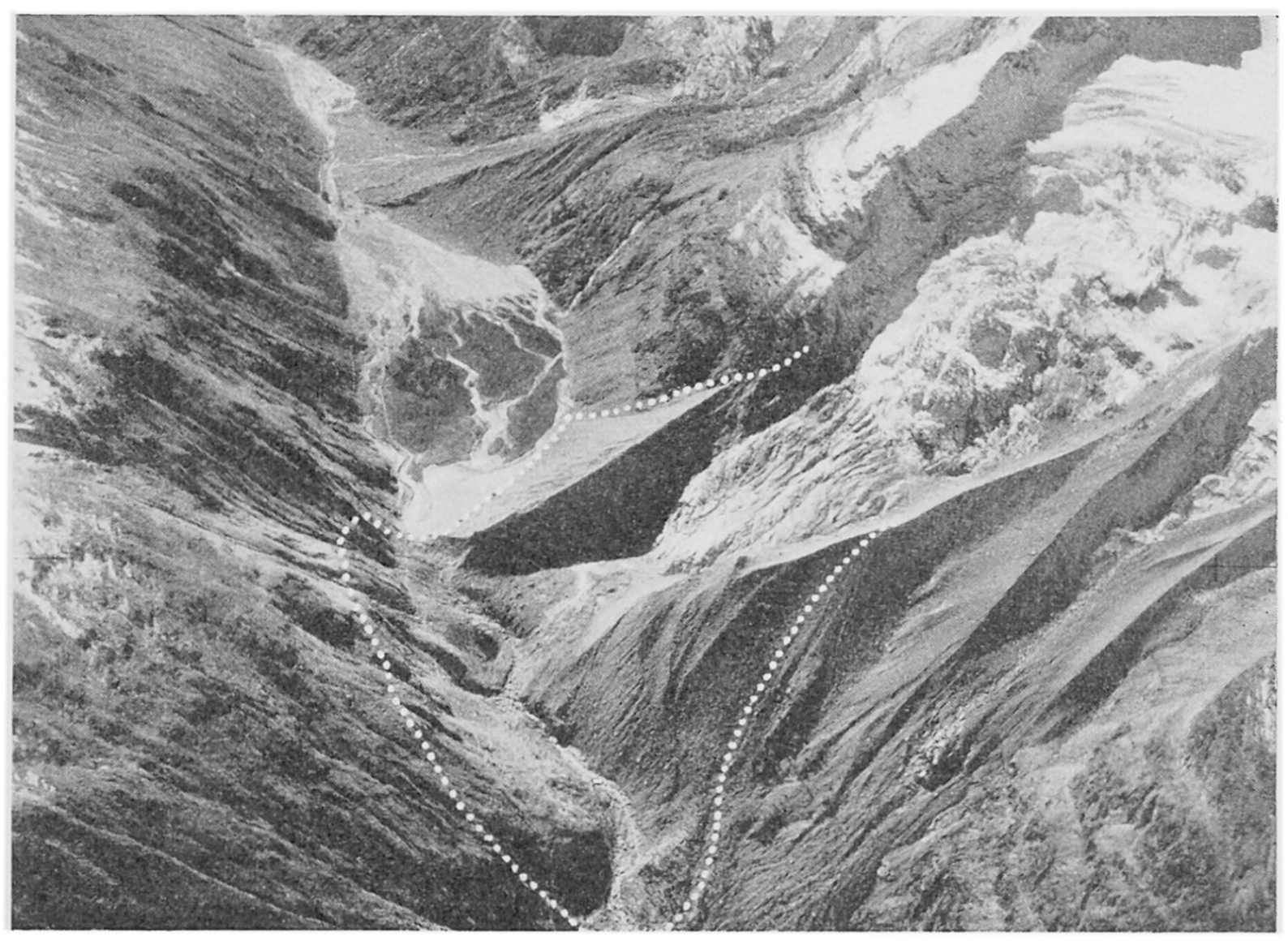

Abb. 4 Luftaufnahme der Ebene von Mattmark und des Allalingletschers. Blick nach Süden (talaufwärts). Die punktierte Linie zeigt den Maximalstand des Gletschers im Jahre 1825 Photo Militärflugdienst 5.11 .1954 


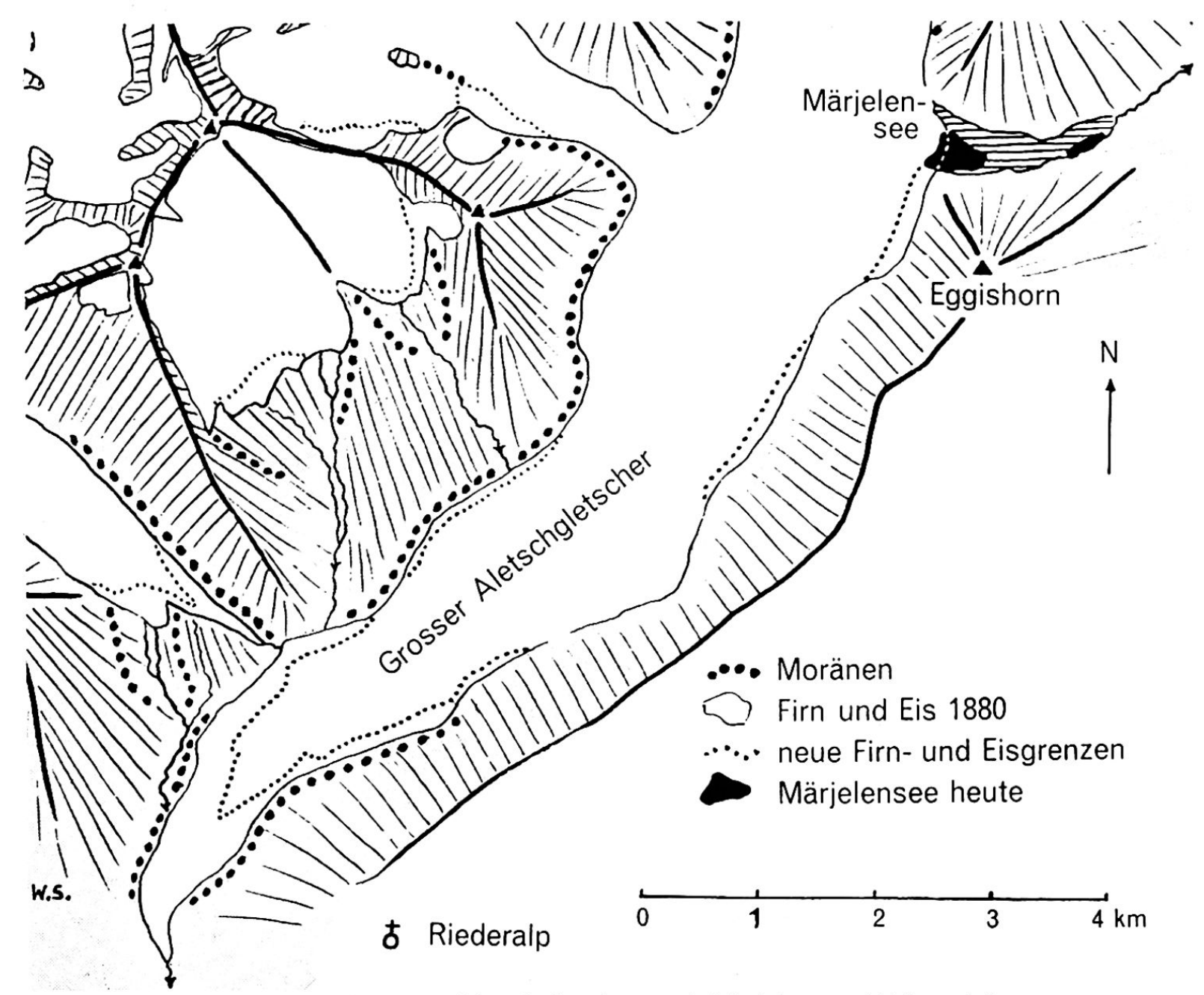

Abb. 5 Aletschgletscher und Märjelensee 1880 und heute

schüttungsebene zur Folge haben, wäre die Bezeichnung «Typ Rofen» ${ }^{4}$ geeignet. Sie paßt vor allem für Gletscher mit ganz geringer Moränenfracht.

Einen andern Typ stellen diejenigen Seelein dar, die sich an der Vereinigung zweier Gletscher bilden können, und zwar oft in Form eines Dreiecks zwischen dem Berg und den beiden Gletscherflanken. Nach dem bekannten Gornersee zwischen dem Monte Rosa- und dem Gornergletscher kann dafür der Name «Typ Gorner» dienen. Im Mont Blanc-Gebiet findet sich eine analoge Erscheinung zwischen dem Glacier du Tacul und dem Glacier de Leschaux. Vom Gornersee, wie auch vom Lac du Tacul sind wiederholte Entleerungen durch Gletscherspalten bekannt.

Als letzte Art von Randseen ist der «Typ Märjelen»zu nennen. Bild 5 zeigt, da $\beta$ es sich um die Stauwirkung eines Haupttalgletschers handelt, die das Niederschlags- und Schmelzwasser in einem kleinen Seitental zurückhält. Der Märjelensee ist eingehend untersucht worden (Lit. 7) : es sei daraus hervorgehoben, daß sein Abfluß meist durch Gletscherspalten erfolgt, gelegentlich auch durch Überlauf über den Aletschgletscher oder, im letzten Jahrhundert, durch Überlauf ins Fieschertal. Recht häufig traten rasche Entleerungen des Sees durch Gletscherspalten ein, z. B. am 19. Juli 1878, als der See beim höchsten Stand innert neun Stunden unter Krachen und Gurgeln im Gletscher rund neun Mill. $\mathrm{m}^{3}$ Wasser verlor. Die Massa führte das Hochwasser zur Rhone, an welcher bei Sion ein Anstieg um 1,5 m beobachtet wurde. Heute ist der Märjelensee sehr viel kleiner, weil die stauende Eiswand des Aletschgletschers infolge Ablation und verringertem Eisnachschub an Mächtigkeit verloren hat.

4 Der Vernagtgletscher mit dem Rofensee ist von Finsterwalder, Hess, Richter u. a. eingehend beschrieben worden. 
Zahlreich sind die Fälle aller Arten von Randseen, die aus den vergletscherten Gebieten aller Erdteile gemeldet werden. Besondere Größe erreichten Stauseen im Karakorum, in Süd- und Nordamerika, bei deren Ausbrüchen stärkste Verheerungen eintraten (Lit. 1, 5, 6 und 9).

Schließlich sei noch auf die subglazialen und intraglazialen Wasseransammlungen hingewiesen, die meist anfänglich unbemerkt blieben und sich erst beim unerwarteten Auslaufen mit Hochwasser der Gletscherbäche manifestierten. Genau untersucht wurden die Höhlungen im Eis der Tête Rousse in $3100 \mathrm{~m}$ Höhe, aus denen am 12. Juli 1892 etwa $100000 \mathrm{~m}^{3}$ Wasser ausbrachen, um in einem furchtbaren Strom mit Eis und Geröll das Dorf Bionnay und die Bäder von St-Gervais zu zerstören. Ebenfalls unter dem Eis verborgen waren rund 11\%2 Mill. Kubikmeter Wasser im Glacier de Ferpècle und im Glacier du Mont Miné, welche im August 1943 eine Hochflut der Borgne veranlaßten.

Abschließend sei versucht, die Gletscherseen in ein System zu fassen, das ihre Verwandtschaft sowie ihre Merkmale zum Ausdruck bringt.

Randseen

\begin{tabular}{|c|c|c|}
\hline Bezeichnung & gestaut durch & Abfluß \\
\hline Typ Cohup & Endmoräne & $\begin{array}{l}\text { Überlauf } \\
\text { Ausbruch einmalig }\end{array}$ \\
\hline Typ Mattmark & $\begin{array}{l}\text { Moräne und } \\
\text { Zunge des } \\
\text { Seitengletschers }\end{array}$ & $\begin{array}{l}\text { Überlauf und sub- } \\
\text { glazial, Ausbrüche } \\
\text { wiederholt }\end{array}$ \\
\hline Typ Rofen & $\begin{array}{l}\text { Normale oder } \\
\text { regenerierte } Z \text { unge } \\
\text { des Seitengletschers }\end{array}$ & $\begin{array}{l}\text { subglazial oder } \\
\text { durch Spalten } \\
\text { Ausbr. wiederholt }\end{array}$ \\
\hline Typ Gorner & $\begin{array}{l}\text { Eis und Moräne } \\
\text { von zwei sich } \\
\text { vereinigenden } \\
\text { Gletschern }\end{array}$ & $\begin{array}{l}\text { durch Spalten; } \\
\text { rasche Entleerung } \\
\text { kommt vor }\end{array}$ \\
\hline Тур Märjelen & $\begin{array}{l}\text { Eis des Gletschers } \\
\text { im Haupttal }\end{array}$ & $\begin{array}{l}\text { Überlauf od. durch } \\
\text { Spalten; oft rasche } \\
\text { Entleerung }\end{array}$ \\
\hline Eisseen & $\begin{array}{l}\text { Gletschereis } \\
\text { (Oberfläche) }\end{array}$ & $\begin{array}{l}\text { Überlauf und } \\
\text { durch Spalten }\end{array}$ \\
\hline cerba & Gletschereis & $\begin{array}{l}\text { durch Spalten; } \\
\text { Ausbrüche }\end{array}$ \\
\hline
\end{tabular}

spätere Form oder Anzeichen

Beispiele Laguna Cohup, Steingletscher Mattmarksee, Lac Combal, Laguna Parrón Vernagtgletscher mit Rofensee, Giétrozgletscher Gornersee, Lac du Tacul

Märjelensee, Rutorsee, zeitw. See am Otemmagletscher ephemere Bildung

Tête Rousse, Ferpèclegletscher durchbrochene

Endmoräne

Ebene hinter der Moräne

event. lokale Ablagerung von Sand oder Glazialton

event. Gletschermühlen oder -dolinen

Die vorstehende Darstellung verschiedenartiger Gletscheseen weist auf dieses Phänomen hin im Hinblick auf eine wünschbare umfassende Bearbeitung, z. B. im Rahmen von Forschungen des Int. Geophysikalischen Jahres.

\section{LITERATUR}

1) Drygalski E. und Machatcheck F.: Gletscherkunde. Wien 1942. - 2) Heim Alb.: Handbuch der Gletscherkunde, Stuttgart 1885. - 3) Heim AlB.: Geologie der Schweiz Bd. I Leipzig 1919. - 4) Heim, Arnold : Wunderland Peru. Bern 1948. - 5) Hess H.: Die Gletscher. Braunschweig 1904. - 6) Klebelsberg R. v.: Handbuch der Gletscherkunde. Wien 1948. - 7) LüTschg O.: Der Märjelensee. Bern 1915. - 8) LüTschg O.: Niederschlag und Abfluß im Hochgebirge. Zürich 1926. - 9) RABOT C.: Les Débâcles glaciaires. Bull. de Géographie hist. et descriptive $\mathrm{N}^{\circ} 3$, Paris 1905. - 10) TöNDURY G. A.: Ursachen und Bekämpfungsmöglichkeiten der zunehmenden Hochwassergefahr im Engadin. Wasser- und Energiewirtschaft Nr. 12. Zürich 1954.

\section{GLACIER-LAKES}

A description is given of the outbreak of the melting-water-basin at the end of the SteinGlacier near the Sustenpass (Switzerland) which happened on the 29th of July 1956. This kind of lake is named "Type Cohup" after the famous Laguna Cohup in the glaciated mountains of Peru. Other examples, situated in the Swiss and Austrian Alps, serve to classify the lakes boarding a Glacier under the names of "Type Mattmark", "Type Rofen", "Type Gorner" and "Type Märjelen" depending on their situation and relation to the glacier. In conclusion some further words inform about lakes of melting water on the ice and about accumulations of water in the interior of glaciers and their behaviour. 